\title{
Crystal Structure Features of $\mathrm{CsPbBr}_{3}$ Perovskite Prepared by Mechanochemical Synthesis
}

\author{
Carlos A. López,* Carmen Abia, María Consuelo Alvarez-Galván, Bo-Kyung Hong, \\ M. Victoria Martínez-Huerta, Federico Serrano-Sánchez, Felix Carrascoso, Andrés Castellanos-Gómez, \\ M. Teresa Fernández-Díaz, and José A. Alonso*
}

Cite This: ACS Omega 2020, 5, 5931-5938

Read Online

ACCESS | 네 Metrics \& More | 回 Article Recommendations | st Supporting Information

ABSTRACT: We present a mechanochemical procedure, with solvent-free, greenchemistry credentials, to grow all-inorganic $\mathrm{CsPbBr}_{3}$ perovskite. The crystal structure of this perovskite and its correlations with the physicochemical properties have been studied. Synchrotron X-ray diffraction (SXRD) and neutron powder diffraction (NPD) allowed us to follow the crystallographic behavior from 4 to $773 \mathrm{~K}$. Unreported features like the observed negative thermal expansion of the $b$ unit-cell parameter stem from octahedral distortions in the 4-100 K temperature range. The mechanochemical synthesis was designed to reduce the impact energy during the milling process, leading to a defect-free, well-crystallized sample characterized by a minimum unit-cell volume and octahedral tilting angles in the low-temperature orthorhombic perovskite framework, defined in the Pbnm space group. The UV-vis diffuse reflectance spectrum shows a reduced band gap of $2.22(3) \mathrm{eV}$, and the photocurrent characterization in a photodetector reveals excellent properties with potential applications of this material in optoelectronic devices.

\section{INTRODUCTION}

All-inorganic cesium lead halide perovskites $\left(\mathrm{CsPbX}_{3}, \mathrm{X}=\mathrm{I}\right.$, $\mathrm{Br}$, and $\mathrm{Cl}$ ) have attracted widespread attention because of their improved stability and balanced carrier mobility compared with their hybrid organic-inorganic counterparts. Nevertheless, the electrical and optical properties of these inorganic perovskites are strongly determined by their compositions, morphologies, and crystallographic phases. ${ }^{1-4}$

Among them, the best candidate for photovoltaic applications is $\mathrm{CsPbI}_{3}$, which shows a low band gap of 1.73 $\mathrm{eV}$ when the cubic phase is preserved. However, bulk $\mathrm{CsPbI}_{3}$ can only maintain the cubic perovskite structure (black phase) above $\approx 593 \mathrm{~K}, 5$ and it transforms into an orthorhombic nonperovskite (yellow phase) material at room temperature, losing its photovoltaic property. The addition of bromide to the halide anion makes the black phase at room temperature more stable owing to the increased effective tolerance factor and a lower phase-transition temperature. ${ }^{1,6}$ However, the larger electronegativity differences between the halogen and lead result in a more ionic bonding character, yielding shorter bond lengths and a larger band gap. This fact limits the shortcircuit current $\left(\mathrm{J}_{\mathrm{SC}}\right)$ of perovskite solar cells (PSCs) but has great potential in tandem and semitransparent photovoltaic applications.

$\mathrm{Cs} \mathrm{PbBr}_{3}$ has attracted much interest because it possesses a stable crystalline structure (orthorhombic phase) at room temperature and, depending on its morphology, it can retain high carrier mobility, good optoelectronic properties, large photoluminescence quantum yield, and superior stability under humidity and thermal attacks. These properties make it suitable for applications in various optoelectronic devices such as light-emitting diodes, photovoltaic cells, photodetectors, and lasers. ${ }^{1,7-11}$

$\mathrm{Cs} \mathrm{PbBr}_{3}$ is usually prepared by reacting equimolar amounts of $\mathrm{CsBr}$ and $\mathrm{PbBr}_{2}$ through conventional wet procedures, while it was described to be prepared through dry methods in only four works. Stoumpos et al. ${ }^{7}$ and Linaburg et al. ${ }^{12}$ used solidstate reactions (milling and heating), while Posudievsky et al. ${ }^{13}$ and $\mathrm{Pal}$ et al. ${ }^{14}$ reported a mechanochemical procedure (without further heating).

In this work, $\mathrm{CsPbBr}_{3}$ was obtained by a mechanosynthesis procedure in a planetary ball mill at room temperature. This synthetic process involves simplicity, swiftness, and reproducibility in line with the green chemistry credentials (e.g., solventless solid-state synthesis). The combination of a moderate mechanical energy generated under mild ball-milling conditions and the inherent chemical modification of

Received: December 11, 2019

Accepted: February 3, 2020

Published: March 10, 2020 
structures/surfaces makes this methodology extremely promising for greener perovskite syntheses, yielding well-crystallized powders with excellent photovoltaic and optoelectronic properties.

\section{EXPERIMENTAL SECTION}

$\mathrm{CsPbBr}_{3}$ was obtained as a microcrystalline powder from mechanosynthesis in a planetary ball mill, from stoichiometric amounts of $\mathrm{CsBr}$ (Strem) and $\mathrm{PbBr}_{2}$ (Alfa Aesar) processed in an $\mathrm{N}_{2}$ atmosphere. A total of $1.5 \mathrm{~g}$ of the reactants was milled using 30 zirconia balls of $5 \mathrm{~mm}$ diameter, with a final mass ratio of 8.6:1, for $4 \mathrm{~h}$ at $400 \mathrm{rpm}$ in a Retsch PM100 mill. A laboratory X-ray diffraction (XRD) pattern was collected on a Bruker D5 diffractometer with $\mathrm{K} \alpha \mathrm{Cu}(\lambda=1.5418 \AA)$ radiation. To study the crystallographic structure, a neutron powder diffraction (NPD) pattern at room temperature (298 K) was collected using the HRPT diffractometer of the SINQ spallation source (PSI, Paul Scherrer Institute, Villigen, Switzerland) with a wavelength of $1.494 \AA$. The crystal structure at lower temperatures was investigated from NPD patterns sequentially collected from 100 to $4 \mathrm{~K}$ in the D20 instrument (Institute Laue Langevin, Grenoble, France) with a wavelength of $1.540 \AA$. The sample, contained in a V cylinder, was introduced in a standard "orange" cryostat and measured at $100 \mathrm{~K}$ for $1 \mathrm{~h}$, and then cooled down to $4 \mathrm{~K}$ while acquiring sequential patterns every $3 \mathrm{~min}$. Finally, a good statistics pattern was collected at $4 \mathrm{~K}$ for $30 \mathrm{~min}$. To investigate the high-temperature structural evolution, synchrotron X-ray powder diffraction (SXRD) patterns were collected at RT, 473, 673, and $773 \mathrm{~K}$ in the MSPD high-resolution diffractometer at the ALBA facility, Barcelona (Spain), selecting an incident beam with $38 \mathrm{keV}$ energy $(\lambda=0.3252$ $\AA)$. The high angular-resolution mode (MAD setup) was selected. ${ }^{15}$ The polycrystalline powder was collected in quartz capillaries of $0.7 \mathrm{~mm}$ diameter, which were kept rotating during the acquisition time. In both cases, the refinement of the structure was performed by the Rietveld method using the Fullprof software. ${ }^{16,17}$ A pseudo-Voigt function was chosen to generate the line shape of the diffraction peaks. The background was interpolated between regions devoid of reflections. The following parameters were refined in the final run: scale factor, background coefficients, zero-point error, pseudo-Voigt corrected for asymmetry parameters, positional coordinates, anisotropic displacement factors, and occupancy factors. For the neutron refinements, the coherent scattering lengths for $\mathrm{Cs}, \mathrm{Pb}$, and $\mathrm{Br}$ were 5.42, 9.405, and $6.795 \mathrm{fm}$, respectively. The scanning electron microscopy (SEM) images were obtained on a Hitachi instrument, model TM-1000. The optical diffuse reflectance spectrum was measured at room temperature using a UV-vis spectrophotometer Varian Cary 5000. The optoelectronic properties of the $\mathrm{CsPbBr}_{3}$ crystals were studied by fabricating a photodetector by drop-casting a $\mathrm{Cs} \mathrm{PbBr}_{3}$ suspension in dimethyl sulfoxide (ratio perovskite/ solvent, 1:4, weight) onto a $\mathrm{SiO}_{2} / \mathrm{Si}$ substrate with prepatterned gold electrodes separated by $10 \mu \mathrm{m}$ (Ossila). The photoresponse was analyzed by illuminating the device with different light-emitting diode (LED) sources with wavelengths ranging from 420 to $1050 \mathrm{~nm}(1.18-2.95 \mathrm{eV})$. The light from the LED sources was focused to form a spot $(400 \mu \mathrm{m}$ in diameter) on the sample, and the intensity was adjusted to achieve a power density of $16 \mathrm{~mW} / \mathrm{cm}^{2}$.

\section{RESULTS AND DISCUSSION}

3.1. Initial Characterization. $\mathrm{CsPbBr}_{3}$ was obtained as a yellowish polycrystalline powder. The initial crystallographic identification of $\mathrm{CsPbBr}_{3}$ was carried out using laboratory XRPD. A Le Bail refinement, illustrated in Figure 1, shows that

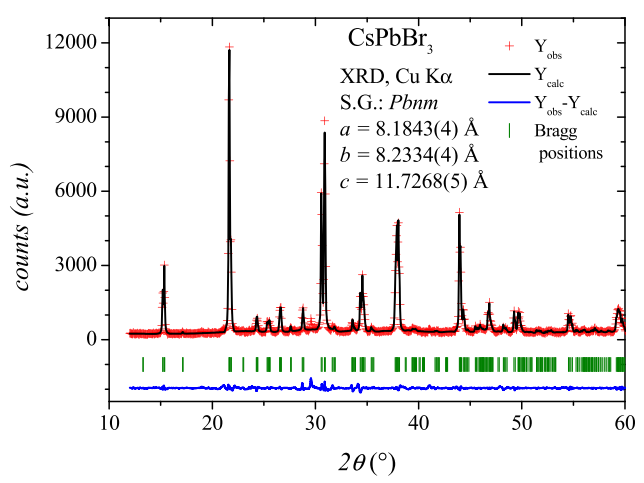

Figure 1. Le Bail fit of a laboratory $\mathrm{XRD}$ pattern of $\mathrm{CsPbBr}$, prepared by ball milling.

$\mathrm{CsPbBr}_{3}$ is pure and presents the characteristic distortion defined in the orthorhombic symmetry, space group Pbnm. The earliest crystal elucidations were made by XRD and NPD in the 1970s. ${ }^{18}$ Recently, several structures have been reported using laboratory and/or synchrotron X-ray diffraction data, ${ }^{9,12-14,19}$ but there are no recent measurements from NPD data.

3.2. Room-Temperature Combined Neutron and Synchrotron X-ray Diffraction Characterization. For a precise crystal structure resolution, both NPD and synchrotron SXRD were combined in a joint refinement; hence, both patterns were modeled in the mentioned $\mathrm{Pbnm}$ space group. The $\mathrm{Cs}^{+}$and $\mathrm{Pb}^{2+}$ cations are located at $4 c(x, y, 1 / 4)$ and $4 b$ $(1 / 2,0,0)$ Wyckoff sites, while $\mathrm{Br} 1$ and $\mathrm{Br} 2$ atoms are placed in $4 c(x, y, 1 / 4)$ and $8 d(x, y, z)$ sites. Figure 2 illustrates the quality of the fit for both NPD and SXRD patterns, including anisotropic refinement of the displacement factors for all the atoms. The figure also includes a view of the crystal structure, highlighting the tilting of the $\mathrm{PbBr}_{6}$ octahedra. Table 1 lists the main crystallographic data. As it is well known, the orthorhombic Pbnm crystal structure in perovskites consists of a three-dimensional (3D) framework of corner-sharing octahedra $\left(\mathrm{PbBr}_{6}\right)$, tilted antiphase along the (100) and (010) directions of the pseudocubic cell and in-phase along the (001) direction, which correspond to $\mathrm{a}^{-} \mathrm{a}^{-} \mathrm{b}^{+}$Glazer's notation as derived by Woodward for a simple perovskite. ${ }^{20,21}$ The tilting angles, estimated as $\varphi=\left(180^{\circ}-\theta\right)$, where $\theta=\langle\mathrm{Pb}-\mathrm{Br}-\mathrm{Pb}\rangle$, are 7.43 and $11.28^{\circ}$ for the antiphase and in-phase tilts at RT, respectively. These compare well with the values of 6.8 and $11.35^{\circ}$ found by Linaburg et al. ${ }^{12}$ for $\mathrm{CsPbBr}_{3}$ at RT.

3.3. Low-Temperature Neutron Diffraction Characterization. Additional NPD patterns were measured at 100 and 4 $\mathrm{K}$; besides, several patterns were sequentially collected during the cooling process. These data reveal that the orthorhombic unit cell is maintained down to $4 \mathrm{~K}$. Figure 3 shows the $a, b$, and $c$ unit-cell parameter variation as well as the Rietveld plot at $4 \mathrm{~K}$. It is remarkable that $a$ and $c$ parameters decrease, whereas $b$ increases upon cooling. This conspicuous effect of negative thermal expansion along the $b$-axis has not been reported before for $\mathrm{CsPbBr}_{3}$. Normally, negative thermal expansion in $\mathrm{Pbnm}$ perovskites is a consequence of magneto- 
(a)

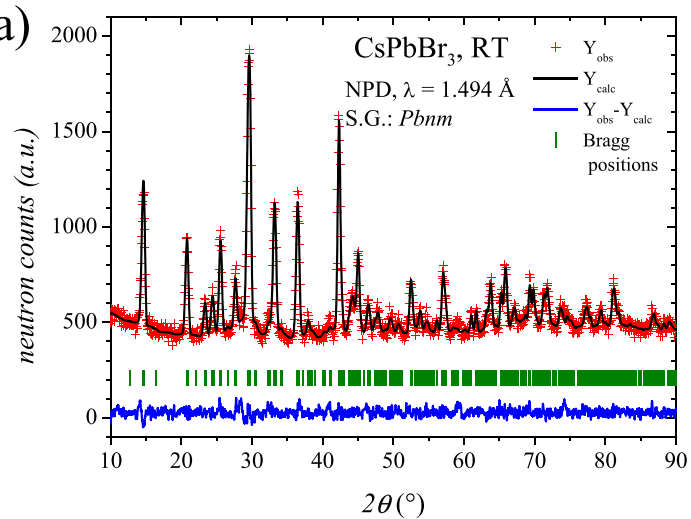

(b)

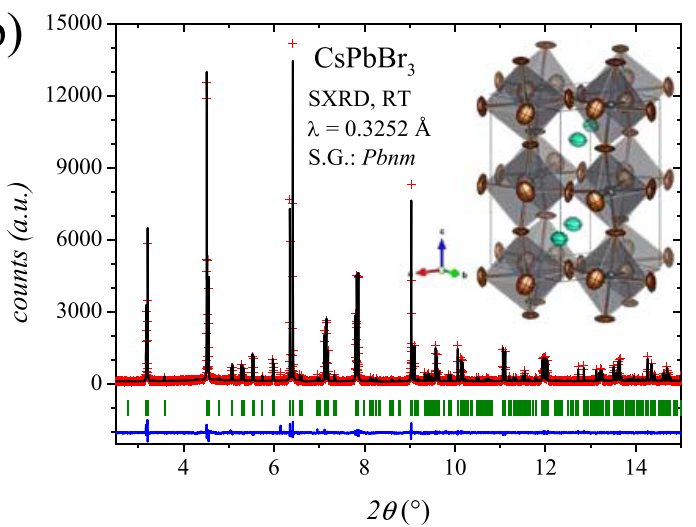

Figure 2. Observed (crosses), calculated (black line), and difference (blue line) profiles after the Rietveld refinement in an orthorhombic cubic unit cell for (a) NPD and (b) SXRD. Inset: view of the crystal structure enhancing the tilting of the $\mathrm{PbBr}_{6}$ octahedra and the anisotropic displacement factors.

Table 1. Crystallographic Data for $\mathrm{CsPbBr}_{3}$ Phase in the Orthorhombic System (Pbnm) from Combined NPD and SXRPD at $\mathrm{RT}^{a}$

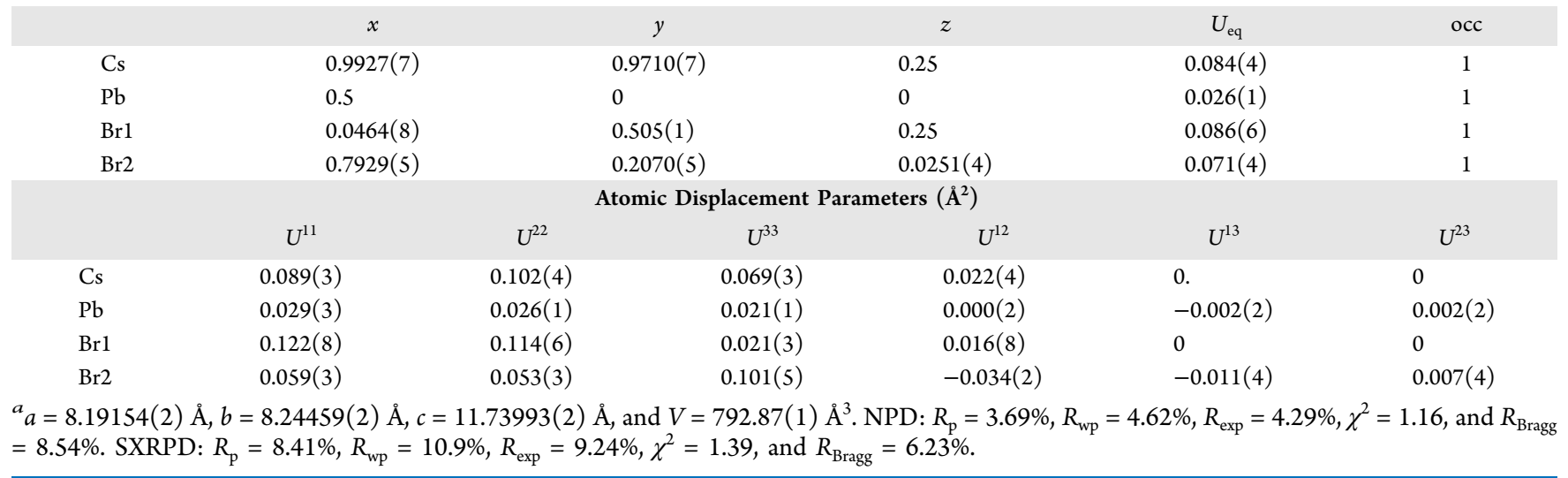

restrictive effects, concomitant with magnetic ordering, for instance in rare-earth ferrites. In $\mathrm{CsPbBr}_{3}$, it deserves further analysis. Table 2 lists the main crystallographic parameters from the pattern collected at $4 \mathrm{~K}$. The Rietveld refinement and the crystallographic data at $100 \mathrm{~K}$ are displayed in Figure S1 and Table S1, respectively. The octahedral tiltings at $4 \mathrm{~K}$ are 10.97 and $14.11^{\circ}$ for antiphase and in-phase tilts, respectively. The thermal evolution (including the RT and the range between 100 and $4 \mathrm{~K}$ ) of the tilts shows a linear behavior for both phase and antiphase octahedral rotations.

The unit-cell volume evolution is plotted in Figure 4a, which shows a constant reduction to reach a plateau below $20 \mathrm{~K}$; this can be a compromise between the contractions of $a$ and $c$ and the expansion observed in $b$. A subsequent reduction is observed close to $4 \mathrm{~K}$. Hence, other structural parameters such as the atomic displacement parameters (ADPs) and interatomic distances were examined as a function of temperature, showing that the $\langle\mathrm{Cs}-\mathrm{Br}\rangle$ distance exhibits a monotonic contraction, while the $\langle\mathrm{Pb}-\mathrm{Br}\rangle$ distances in the $\mathrm{PbBr}_{6}$ octahedra remain unaltered within the experimental errors (Figure 4b). This thermal evolution is reasonable considering the greater covalent component existing between $\mathrm{Pb}-\mathrm{Br}$ with respect to $\mathrm{Cs}-\mathrm{Br}$ interactions, implying that the unit-cell volume contraction stems from the rotation of quasirigid octahedra. Second, the ADPs exhibit a conventional reduction for $\mathrm{Cs}^{+}$ions, whereas they remain almost constant for $\mathrm{Pb}^{2+}$; this observation can be a symptom of the much more covalent $\mathrm{Pb}-\mathrm{Br}$ and rigid bond that locks the $\mathrm{Pb}$ displacement factors. This behavior close to $4 \mathrm{~K}$ can be driven by a structural limitation in the $\mathrm{PbBr}_{6}$ octahedral framework to follow the progressive reduction of the $\mathrm{Cs}-\mathrm{Br}$ bonds, leading to octahedral distortions that account for the expansion of parameter $b$.

3.4. High-Temperature Synchrotron X-ray Diffraction Characterization. For the high-temperature analysis, an SXRD experiment was performed at selected temperatures (473, 673, and $773 \mathrm{~K})$. As mentioned above, the RT pattern confirms the already described orthorhombic symmetry; however, at $473 \mathrm{~K}$ and above, the structure can be defined in the cubic $P m \overline{3} m$ space group. Figure 5 shows a selected angular range that illustrates this phase transition. It is important to remark that between the orthorhombic and cubic phases (RT to $473 \mathrm{~K}$ ) a transient tetragonal $(\mathrm{P} 4 / \mathrm{mbm}$ ) phase has been previously reported, ${ }^{7,18}$ which we could not identify.

At $473 \mathrm{~K}$ and above, the cubic symmetry is defined in the space group $P m \overline{3} m$. Cs atoms are placed at $1 c(1 / 2,1 / 2,1 / 2)$ Wyckoff site, $\mathrm{Pb}$ at $1 a(0,0,0)$, and $\mathrm{Br}$ at $3 d(1 / 2,0,0)$. Figure 6 plots the Rietveld refinements at different temperatures, showing an excellent agreement between the observed and the calculated profiles, including an inset with a view of the cubic crystal structure above $473 \mathrm{~K}$. The main crystallographic data are listed in Table 3.

The thermal evolution of the unit-cell parameters is illustrated in Figure 7 as volume $/ Z$ versus temperature. On the other hand, the comparison of the unit-cell volume with 

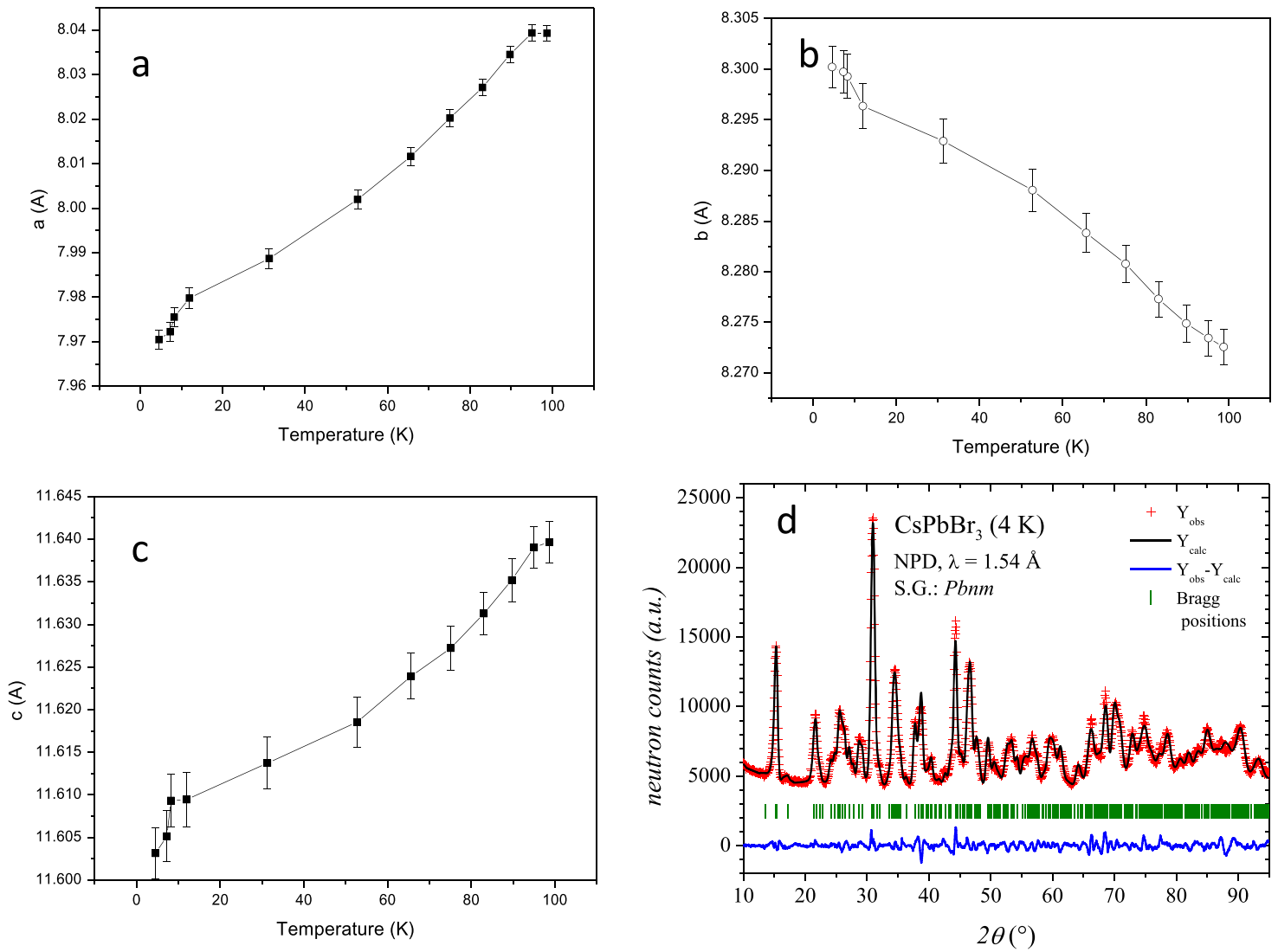

Figure 3. Thermal evolution of (a) $a$, (b) b, and (c) $c$ unit-cell parameters. (d) Rietveld NPD profiles at $4 \mathrm{~K}$.

Table 2. Crystallographic Data for $\mathrm{CsPbBr}_{3}$ Phase in the Orthorhombic System (Pbnm) from NPD at $4 \mathrm{~K}^{a}$

\begin{tabular}{llllll} 
& \multicolumn{1}{c}{$x$} & \multicolumn{1}{c}{$y$} & \multicolumn{1}{c}{$z$} & $U_{\text {iso }}$ & occ \\
$\mathrm{Cs}$ & $0.9800(8)$ & $0.9378(5)$ & 0.25 & $0.021(1)$ & 1 \\
$\mathrm{~Pb}$ & 0.5 & 0 & 0 & $0.015(1)$ & 1 \\
$\mathrm{Br} 1$ & $0.0701(5)$ & $0.5078(6)$ & 0.25 & $0.027(1)$ & 1 \\
$\mathrm{Br} 2$ & $0.8020(4)$ & $0.2007(4)$ & $0.0369(3)$ & $0.019(1)$ & 1
\end{tabular}

$a_{a}=7.9734(7) \AA, b=8.3065(8) \AA, c=11.612(1) \AA$, and $V=$ $769.1(1) \AA^{3} . R_{\mathrm{p}}=2.62 \%, R_{\mathrm{wp}}=3.40 \%, R_{\exp }=1.20 \%, \chi^{2}=8.03$, and $R_{\text {Bragg }}=4.33 \%$.

those reported in the literature reveals significant differences, as illustrated in the inset of Figure 7 . The obtained unit-cell volume is subtly lower than those of the previous reports. Additionally, a correlation with the synthesis method can be established. In general, the samples synthesized from the mechanochemical (MC) method (without heating) present a higher unit-cell volume, while those obtained from the solidstate (SS) reaction (with heating) exhibit a smaller unit-cell size. In contrast, our sample obtained by ball-milling exhibits the lowest unit-cell size, which is discussed below.

These subtle changes can be related to the defects in the crystals, since annealing at moderate temperatures may reduce the defects in the sample, producing quality crystals. Moreover, the sample obtained by Stoumpos et al. ${ }^{7}$ in sealed ampoules at $600{ }^{\circ} \mathrm{C}$ presents a smaller cell than that obtained by Linaburg et al. ${ }^{12}$ in air at $425{ }^{\circ} \mathrm{C}$ for $20 \mathrm{~h}$. In this situation, the unit-cell

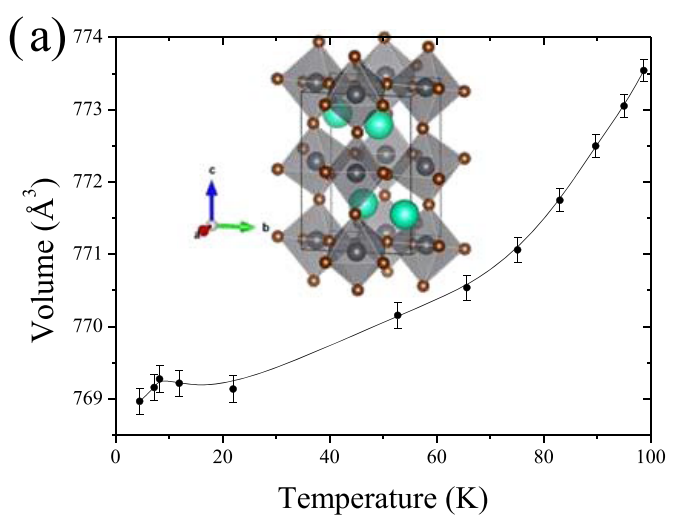

(b)

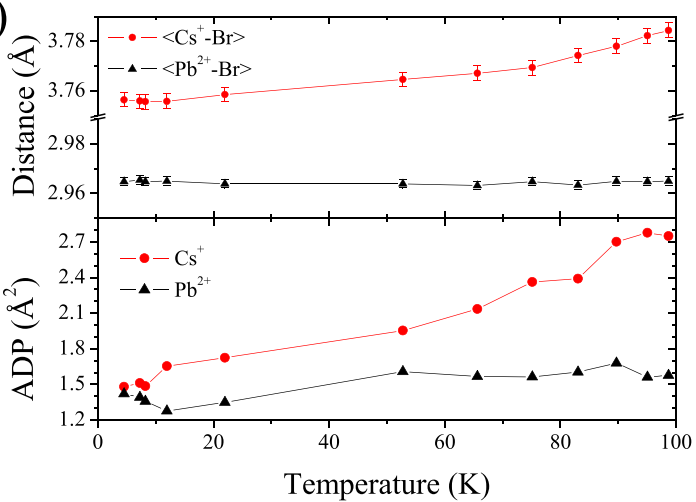

Figure 4. Thermal evolution of the unit-cell volume (a), interatomic distances, and atomic displacement factors (b). Inset: view of the crystal structure at $4 \mathrm{~K}$. 


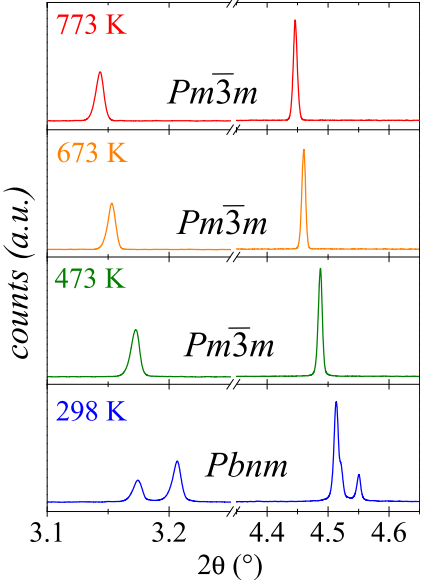

Figure 5. Thermal evolution of selected regions of the SXRD patterns of $\mathrm{CsPBr}_{3}$, in which an orthorhombic to cubic phase transition is evidenced.

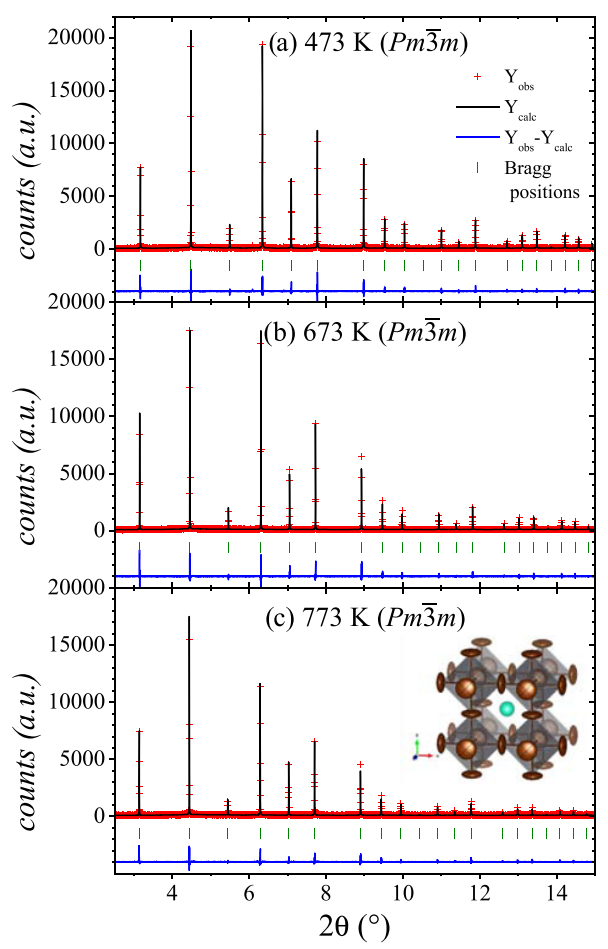

Figure 6. Rietveld refinement of synchrotron XRD patterns at (a) 473 $\mathrm{K}$, (b) $673 \mathrm{~K}$, and (c) $773 \mathrm{~K}$. A view of the cubic crystal structure is given in the inset of $(c)$.

size of the present sample is in the lower limit of the analyzed samples; this fact suggests that the present milling conditions yield a well-crystallized sample with a minimum number of defects. In the mechanosynthesis technique, the energy transferred to the mixture is a determining factor of the synthetic procedure. It depends on the different characteristics of the milling process, such as the balls/mixture mass ratio, grinding time, and rotation speed. These determine the crystallinity and defective nature of the perovskite structure and thus the lattice parameter and the unit-cell volume. If we compare our milling conditions, ball:mixture mass ratio, milling time, and rpm (8.6:1 mass ratio, 30 balls of $5 \mathrm{~mm}$ diameter, for $4 \mathrm{~h}$ at $400 \mathrm{rpm}$ ) with those described by Posudievsky et al. ${ }^{13}$ (29:1 mass ratio, 30 balls of $10 \mathrm{~mm}$
Table 3. Crystallographic Data for $\mathrm{CsPbBr}_{3}$ Phase in the Cubic System $(P m \overline{3} m)$ from SXRPD at High Temperature

\begin{tabular}{llll} 
& \multicolumn{1}{c}{$473 \mathrm{~K}$} & $573 \mathrm{~K}$ & $673 \mathrm{~K}$ \\
& \multicolumn{1}{c}{ Unit Cell } & & \\
$\mathrm{a}(\AA)$ & $5.87330(3)$ & $5.91018(6)$ & $5.92805(6)$ \\
$V\left(\AA^{3}\right)$ & $202.603(2)$ & $206.444(3)$ & $208.322(4)$ \\
$\mathrm{Cs}(0.5,0.5,0.5)$ & & \\
$\quad U_{11}=U_{22}=U_{33}$ & $0.013(2)$ & $0.175(5)$ & $0.198(5)$ \\
$\mathrm{Pb}(0,0,0)$ & & & \\
$\quad U_{11}=U_{22}=U_{33}$ & $0.0383(8)$ & $0.058(1)$ & $0.071(2)$ \\
$\mathrm{Br}(0.5,0,0)$ & & & \\
$\quad U_{11}$ & $0.037(4)$ & $0.054(6)$ & $0.067(6)$ \\
$U_{22}=U_{33}$ & $0.234(5)$ & $0.270(8)$ & $0.292(8)$ \\
$\operatorname{Reliability~Factors~}$ & & & 11.6 \\
$R_{\mathrm{p}}(\%)$ & 10.3 & 11.5 & 16.0 \\
$R_{\text {wp }}(\%)$ & 13.3 & 16.0 & 10.6 \\
$R_{\text {exp }}(\%)$ & 9.82 & 10.5 & 2.26 \\
$\chi^{2}$ & 1.85 & 2.35 & 8.97 \\
$R_{\text {Bragg }}(\%)$ & 3.59 & 8.18 & \\
\hline
\end{tabular}

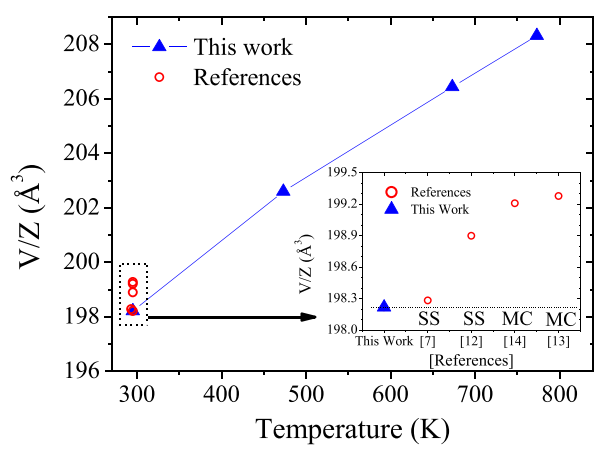

Figure 7. Thermal evolution of the normalized unit-cell volume of $\mathrm{Cs} \mathrm{PbBr}_{3}$ obtained by ball milling compared with other literature values from alternative synthesis techniques.

diameter, for $4 \mathrm{~h}$ at $500 \mathrm{rpm}$ ), the latter are by far more energetic than those used in the present work, thus leading to a more defective material. Moreover, this can be quantified by estimating the ball-impact energy $\left(\Delta E_{\mathrm{b}}\right)$ and weightnormalized cumulative kinetic energy $\left(E_{\text {cum }}\right)$. For the conditions of the present work, the estimated $\Delta E_{\mathrm{b}}$ and $E_{\text {cum }}$ are $4.1 \mathrm{~mJ} / \mathrm{impact}$ and $149 \mathrm{~kJ} / \mathrm{g}$, respectively. However, for Posudievsky et al. ${ }^{13} \Delta E_{\mathrm{b}}$ and $E_{\text {cum }}$ are $20.4 \mathrm{~mJ} /$ impact and 826 $\mathrm{kJ} / \mathrm{g}$, respectively. The kinetic energy given to the sample per impact is approximately 5 times smaller in the present synthesis; hence, it is possible to infer that such moderate conditions for long times yield a better-crystallized, more defect-free sample. We have prepared, therefore, $\mathrm{CsPbBr}_{3}$ perovskite in mild milling conditions, beyond those typically used in literature.

3.5. Microstructure by Scanning Electron Microscopy (SEM). Figure 8 illustrates some typical views of the asprepared $\mathrm{CsPbBr}_{3}$ polycrystals. From a mechanosynthesis process, involving the collision of high-energy $\mathrm{ZrO}_{2}$ balls with the specimens, one would expect a highly disaggregated product formed of small particles. However, surprisingly, we can observe a heterogeneous picture where quite large particles $(10-20 \mu \mathrm{m})$ are mixed up with smaller fragments of undefined shapes (Figure 8a). However, in a larger magnification picture (Figure 8b), it is evident that each particle is indeed formed by an agglomeration of much smaller grains of typically $0.5-1$ 

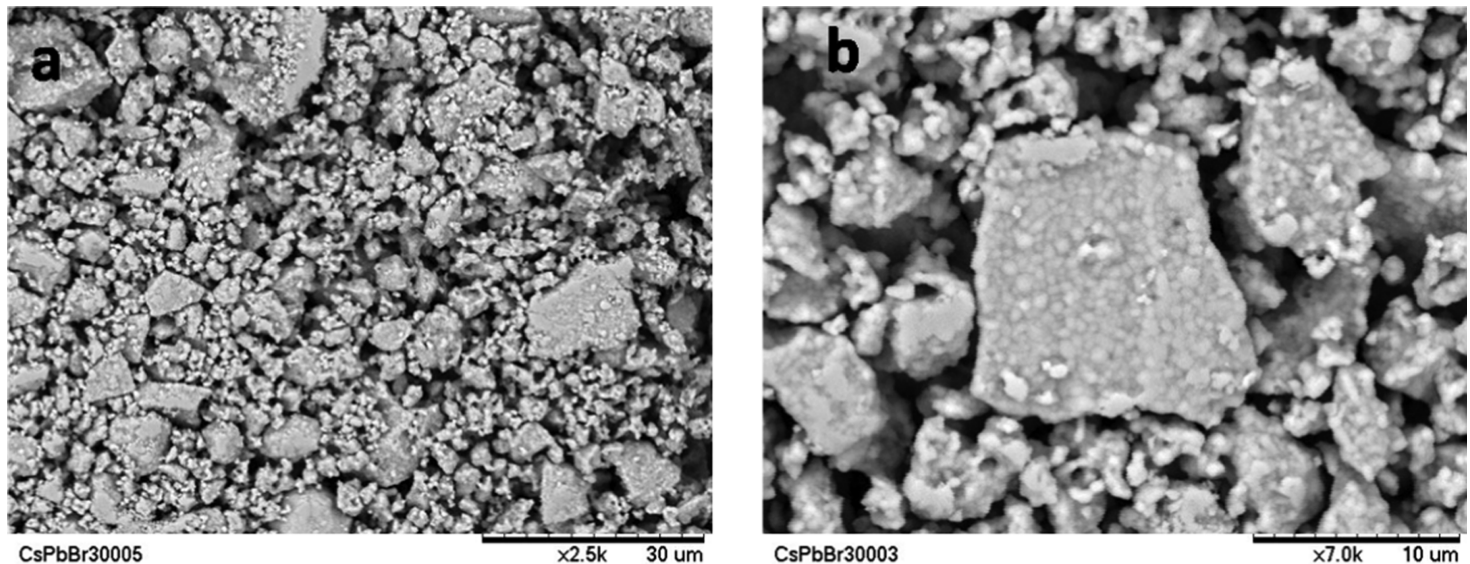

Figure 8. SEM images of $\mathrm{CsPbBr}_{3}$ samples at $2500 \times$ (a) and $7000 \times(\mathrm{b})$ magnifications.

$\mu \mathrm{m}$. We assume that these individual grains are monocrystalline, providing a sufficiently large diffraction domain that accounts for the good crystallinity of the sample versus neutron and X-ray synchrotron diffraction techniques. Altogether, this scenario illustrates that the growth of microcrystals is not perturbed by the dynamical motion of the reactants and $\mathrm{ZrO}_{2}$ balls after $4 \mathrm{~h}$ of reaction. This morphological evidence is in agreement with that previously deduced from the synthesis conditions in terms of $\Delta E_{\mathrm{b}}$ and $E_{\text {cum }}$ energies.

3.6. UV-vis-NIR Spectra. The absorption ability of $\mathrm{CsPbBr}$ powder was determined by diffuse reflectance UVvis spectroscopy. Figure 9 depicts the optical absorption

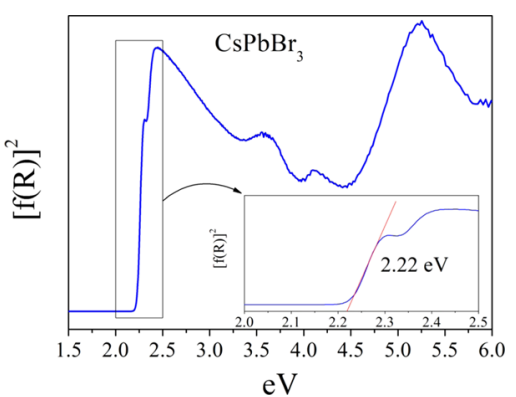

Figure 9. Kubelka-Munk (KM) transformed diffuse reflectance spectrum of $\mathrm{CsPbBr}_{3}$. The inset shows an expanded zone of the absorption edge. coefficient related to the Kubelka-Munk function $(F(R)=a=$ $(1-R) 2 / 2 R, R$ is the reflectance) versus the wavelength in electronvolts. The band gap was calculated by extrapolating the linear region to the abscissa. The value obtained for $\mathrm{CsPbBr}_{3}$ $(\sim 2.22(3) \mathrm{eV})$ is in agreement with data reported in the literature for its band gap at room temperature. ${ }^{6,8,9}$ Moreover, there is a subtle reduction with respect to the value of $2.27 \mathrm{eV}$ given by Linaburg et al. ${ }^{12}$ for a sample prepared by solid-state reactions, which is convenient for use in solar cells.

3.7. Optoelectronic Characterization. Figure 10a shows the time evolution of the current flowing through the device (with a bias voltage of $1 \mathrm{~V}$ applied between electrodes), while the illumination is switched on and off. This measurement allows one to determine the photocurrent (subtracting the current in the dark to the current upon illumination), as well as the response time of the photodetector device. By employing a wavelength of $420 \mathrm{~nm}$, we obtain a photocurrent of $90 \mathrm{nA}$ with response times of 170 and $90 \mathrm{~ms}$ for the rise and decay processes, respectively. In addition, a sizeable overshoot can be clearly seen in this figure, which has already been observed for these materials and has been attributed to a sudden generation of photogenerated charge carriers followed by a slow photocurrent decay toward a steady state when the equilibrium between the charge diffusion rate and its generation rate is achieved. $^{22-24}$
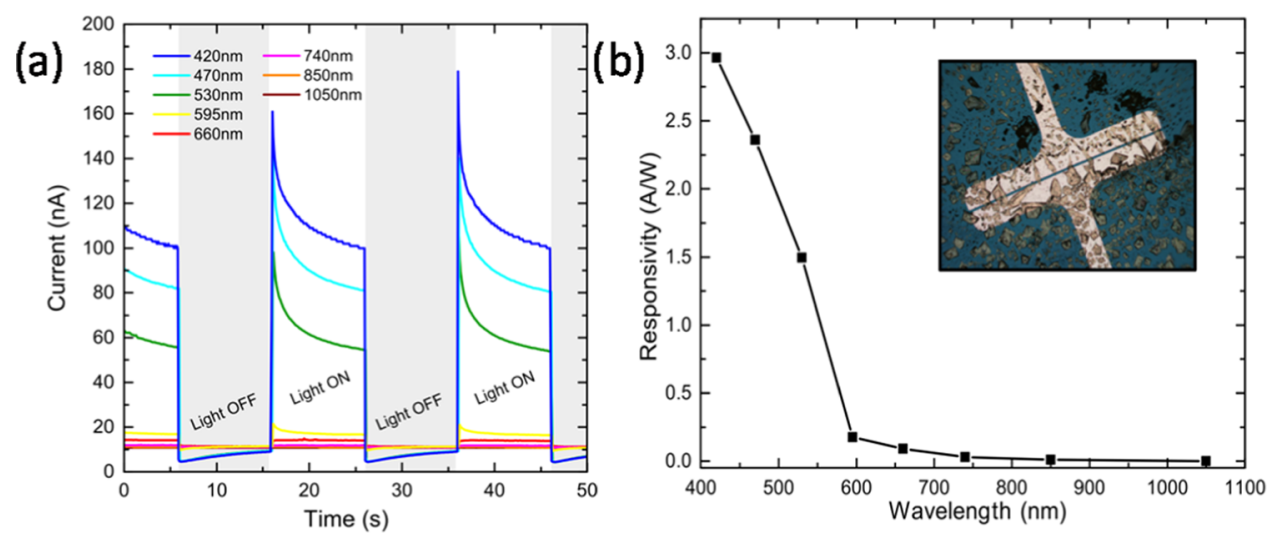

Figure 10. (a) Time evolution of the current flowing through the photodetector (bias of $1 \mathrm{~V}$ ) under alternating dark and light illumination with different wavelengths (power density of $16 \mathrm{~mW} / \mathrm{cm}^{2}$ ). (b) Responsivity of the device as a function of the LED wavelength (bias of $1 \mathrm{~V}$ and power density of $\left.16 \mathrm{~mW} / \mathrm{cm}^{2}\right)$. The inset shows the device investigated. 
To determine the spectral response of our device, we extract the responsivity at different illumination wavelengths. The responsivity is a figure of merit that allows for a direct comparison between different photodetectors. This value is defined as $R=I_{\mathrm{ph}} / P$, where $I_{\mathrm{ph}}$ is the photocurrent and $P$ the effective power, which is given by $P=P_{\text {light }} \cdot A_{\text {dev }} / A_{\text {spot }}$, where $P_{\text {light }}$ is the LED power, $A_{\mathrm{dev}}$ is the area of the material covering the channel of the device and $A_{\text {spot }}$ is the area of the spot.

Figure $10 \mathrm{~b}$ exhibits the responsivity as a function of the illumination wavelength. The responsivity increases at shorter wavelengths, reaching a value of $3 \mathrm{~A} / \mathrm{W}$ at $420 \mathrm{~nm}$, which is higher than that reported, under similar illumination and biasing conditions, for other perovskites like $\mathrm{CsPbCl}_{3}$ (less than $0.5 \mathrm{~A} / \mathrm{W}$ ) or $\mathrm{CsPbI}_{3}$ (less than $0.4 \mathrm{~A} / \mathrm{W}$ ). ${ }^{2,26}$ For $\mathrm{CsPbBr}_{3}$ photodetectors in the literature, the reported responsivity ranges from $\sim 0.005^{24}$ to $\sim 10 \mathrm{~A} / \mathrm{W}^{27}$ and thus our device is in the upper bound of this range.

\section{CONCLUSIONS}

We have obtained a well-crystallized $\mathrm{Cs} \mathrm{PbBr}_{3}$ perovskite from a mechanochemical method under mild milling conditions. The unit-cell parameters at RT were smaller than those described for samples synthesized by ball milling, but they were similar to those of the specimens previously obtained from conventional solid-state reaction methods. The crystallographic features were analyzed from synchrotron X-ray and neutron powder diffraction in the $4-773 \mathrm{~K}$ temperature range. At RT, an orthorhombic superstructure of perovskite, defined in the Pbnm space group, is observed, which becomes cubic $P m \overline{3} m$ above $473 \mathrm{~K}$, in agreement with previous reports. At lower temperatures, the phase remains orthorhombic down to $4 \mathrm{~K}$, and the collapse of the unit cell upon cooling is related to the progressive octahedral tilting; below $8 \mathrm{~K}$, the structure distortion seems to reach a limit in the octahedral rotation. Finally, the optoelectronic properties of our $\mathrm{CsPbBr}_{3}$ specimen, implemented in a photodetector device, demonstrate a high and selective responsivity of $3 \mathrm{~A} / \mathrm{W}$ at shorter wavelengths, improving the reported responsivity ranges.

\section{ASSOCIATED CONTENT}

\section{SI Supporting Information}

The Supporting Information is available free of charge at https://pubs.acs.org/doi/10.1021/acsomega.9b04248.

Observed, calculated, and difference NPD profiles after the Rietveld refinement at $100 \mathrm{~K}$; crystallographic data for $\mathrm{CsPbBr}_{3}$ phase in the orthorhombic system (PDF)

\section{AUTHOR INFORMATION}

\section{Corresponding Authors}

Carlos A. López - Instituto de Ciencia de Materiales de Madrid, CSIC, 28049 Madrid, Spain; INTEQUI, (UNSL-CONICET) and Facultad de Quimica, Bioquimica y Farmacia, UNSL, 5700 San Luis, Argentina; Email: calopez@unsl.edu.com

José A. Alonso - Instituto de Ciencia de Materiales de Madrid, CSIC, 28049 Madrid, Spain; 이이.org/0000-0001-53291225; Email: ja.alonso@icmm.csic.es

\section{Authors}

Carmen Abia - Instituto de Ciencia de Materiales de Madrid, CSIC, 28049 Madrid, Spain; Institut Laue Langevin, 38042 Grenoble Cedex, France
María Consuelo Alvarez-Galván - Instituto de Catálisis y Petroleoquimica, CSIC, 28049 Madrid, Spain

Bo-Kyung Hong - Instituto de Ciencia de Materiales de Madrid and Instituto de Catálisis y Petroleoquimica, CSIC, 28049 Madrid, Spain

M. Victoria Martínez-Huerta - Instituto de Catálisis y Petroleoquimica, CSIC, 28049 Madrid, Spain

Federico Serrano-Sánchez - Instituto de Ciencia de Materiales de Madrid, CSIC, 28049 Madrid, Spain

Felix Carrascoso - Instituto de Ciencia de Materiales de Madrid, CSIC, 28049 Madrid, Spain

Andrés Castellanos-Gómez - Instituto de Ciencia de Materiales de Madrid, CSIC, 28049 Madrid, Spain; (1) orcid.org/0000-0002-3384-3405

M. Teresa Fernández-Díaz - Institut Laue Langevin, 38042 Grenoble Cedex, France

Complete contact information is available at:

https://pubs.acs.org/10.1021/acsomega.9b04248

\section{Notes}

The authors declare no competing financial interest.

\section{ACKNOWLEDGMENTS}

The authors acknowledge the financial support given by the Spanish Ministry of Economy and Competitiveness (ENE2014-52158-C2-1-R project and MAT2017-84496-R) co-founded by FEDER. C.A.L. acknowledges ANPCyT and UNSL for financial support (Projects PICT2017-1842 and PROICO 2-2016), Argentina.

\section{REFERENCES}

(1) Deng, J.; Li, J.; Yang, Z.; Wang, M. All-Inorganic Lead Halide Perovskites: A Promising Choice for Photovoltaics and Detectors. J. Mater. Chem. C 2019, 7, 12415-12440.

(2) Gao, Y.; Wu, Y.; Lu, H.; Chen, C.; Liu, Y.; Bai, X.; Yang, L.; Yu, W. W.; Dai, Q.; Zhang, Y. CsPbBr 3 Perovskite Nanoparticles as Additive for Environmentally Stable Perovskite Solar Cells with 20.46\% Efficiency. Nano Energy 2019, 59, 517-526.

(3) Liang, J.; Wang, C.; Wang, Y.; Xu, Z.; Lu, Z.; Ma, Y.; Zhu, H.; Hu, Y.; Xiao, C.; Yi, X.; et al. All-Inorganic Perovskite Solar Cells. J. Am. Chem. Soc. 2016, 138, 15829-15832.

(4) Liang, J.; Han, X.; Yang, J.; Zhang, B.; Fang, Q.; Zhang, J.; Ai, Q.; Ogle, M. M.; Terlier, T.; Martí, A. A.; et al. Defect-EngineeringEnabled High-Efficiency All-Inorganic Perovskite Solar Cells. Adv. Mater. 2019, 31, No. 1903448.

(5) Dastidar, S.; Hawley, C. J.; Dillon, A. D.; Gutierrez-Perez, A. D.; Spanier, J. E.; Fafarman, A. T. Quantitative Phase-Change Thermodynamics and Metastability of Perovskite-Phase Cesium Lead Iodide. J. Phys. Chem. Lett. 2017, 8, 1278-1282.

(6) Sutton, R. J.; Eperon, G. E.; Miranda, L.; Parrott, E. S.; Kamino, B. A.; Patel, J. B.; Hörantner, M. T.; Johnston, M. B.; Haghighirad, A. A.; Moore, D. T.; et al. Bandgap-Tunable Cesium Lead Halide Perovskites with High Thermal Stability for Efficient Solar Cells. Adv. Energy Mater. 2016, 6, No. 1502458.

(7) Stoumpos, C. C.; Malliakas, C. D.; Peters, J. A.; Liu, Z.; Sebastian, M.; Im, J.; Chasapis, T. C.; Wibowo, A. C.; Chung, D. Y.; Freeman, A. J.; et al. Crystal Growth of the Perovskite Semiconductor $\mathrm{CsPbBr}_{3}$ : A New Material for High-Energy Radiation Detection. Cryst. Growth Des. 2013, 13, 2722-2727.

(8) Li, X.; Tan, Y.; Lai, H.; Li, S.; Chen, Y.; Li, S.; Xu, P.; Yang, J. All-Inorganic $\mathrm{CsPbBr}$ Perovskite Solar Cells with $10.45 \%$ Efficiency by Evaporation-Assisted Deposition and Setting Intermediate Energy Levels. ACS Appl. Mater. Interfaces 2019, 11, 29746-29752.

(9) Zhang, H.; Liu, X.; Dong, J.; Yu, H.; Zhou, C.; Zhang, B.; Xu, Y.; Jie, W. Centimeter-Sized Inorganic Lead Halide Perovskite CsPbBr 3 
Crystals Grown by an Improved Solution Method. Cryst. Growth Des.

2017, 17, 6426-6431.

(10) Dirin, D. N.; Cherniukh, I.; Yakunin, S.; Shynkarenko, Y.; Kovalenko, M. V. Solution-Grown $\mathrm{CsPbBr}_{3}$ Perovskite Single Crystals for Photon Detection. Chem. Mater. 2016, 28, 8470-8474.

(11) Ramasamy, P.; Lim, D. H.; Kim, B.; Lee, S. H.; Lee, M. S.; Lee, J. S. All-Inorganic Cesium Lead Halide Perovskite Nanocrystals for Photodetector Applications. Chem. Commun. 2016, 52, 2067-2070.

(12) Linaburg, M. R.; McClure, E. T.; Majher, J. D.; Woodward, P. M. $\mathrm{Cs}_{1-\mathrm{X}} \mathrm{Rb}_{\mathrm{x}} \mathrm{PbCl}_{3}$ and $\mathrm{Cs}_{1-\mathrm{X}} \mathrm{Rb}_{\mathrm{x}} \mathrm{PbBr}_{3}$ Solid Solutions: Understanding Octahedral Tilting in Lead Halide Perovskites. Chem. Mater. 2017, 29, 3507-3514.

(13) Posudievsky, O. Y.; Konoshchuk, N. V.; Karbivskyy, V. L.; Boiko, O. P.; Koshechko, V. G.; Pokhodenko, V. D. Structural and Spectral Characteristics of Mechanochemically Prepared $\mathrm{CsPbBr}_{3}$. Theor. Exp. Chem. 2017, 53, 235-243.

(14) Pal, P.; Saha, S.; Banik, A.; Sarkar, A.; Biswas, K. All-Solid-State Mechanochemical Synthesis and Post-Synthetic Transformation of Inorganic Perovskite-Type Halides. Chem. - Eur. J. 2018, 24, 18111815.

(15) Fauth, F.; Boer, R.; Gil-Ortiz, F.; Popescu, C.; Vallcorba, O.; Peral, I.; Fullà, D.; Benach, J.; Juanhuix, J. The Crystallography Stations at the Alba Synchrotron. Eur. Phys. J. Plus 2015, 130, No. 160.

(16) Rietveld, H. M. A Profile Refinement Method for Nuclear and Magnetic Structures. J. Appl. Crystallogr. 1969, 2, 65-71.

(17) Rodríguez-Carvajal, J. Recent Advances in Magnetic Structure Determination by Neutron Powder Diffraction. Phys. B: Condens. Matter 1993, 192, 55-69.

(18) Hirotsu, S.; Harada, J.; Iizumi, M.; Gesi, K. Structural Phase Transitions in CsPbBr . J. Phys. Soc. Jpn. 1974, 37, 1393-1398.

(19) Liu, F.; Wang, F.; Hansen, K. R.; Zhu, X.-Y. Bimodal Bandgaps in Mixed Cesium Methylammonium Lead Bromide Perovskite Single Crystals. J. Phys. Chem. C 2019, 123, 14865-14870.

(20) Glazer, A. M. The Classification of Tilted Octahedra in Perovskites. Acta Crystallogr., Sect. B: Struct. Crystallogr. Cryst. Chem. 1972, 28, 3384-3392.

(21) Woodward, P. M. Octahedral Tilting in Perovskites. I. Geometrical Considerations. Acta Crystallogr., Sect. B: Struct. Sci. 1997, 53, 32-43.

(22) Ding, J.; Du, S.; Zuo, Z.; Zhao, Y.; Cui, H.; Zhan, X. High Detectivity and Rapid Response in Perovskite $\mathrm{CsPbBr}_{3}$ Single-Crystal Photodetector. J. Phys. Chem. C 2017, 121, 4917-4923.

(23) Liu, D.; Hu, Z.; Hu, W.; Wangyang, P.; Yu, K.; Wen, M.; Zu, Z.; Liu, J.; Wang, M.; Chen, W.; et al. Two-Step Method for Preparing All-Inorganic $\mathrm{CsPbBr} 3$ Perovskite Film and Its Photoelectric Detection Application. Mater. Lett. 2017, 186, 243-246.

(24) Cha, J. H.; Han, J. H.; Yin, W.; Park, C.; Park, Y.; Ahn, T. K.; Cho, J. H.; Jung, D. Y. Photoresponse of $\mathrm{CsPbBr}_{3}$ and $\mathrm{Cs}_{4} \mathrm{PbBr}_{6}$ Perovskite Single Crystals. J. Phys. Chem. Lett. 2017, 8, 565-570.

(25) Zhang, J.; Wang, Q.; Zhang, X.; Jiang, J.; Gao, Z.; Jin, Z.; Liu, S. High-Performance Transparent Ultraviolet Photodetectors Based on Inorganic Perovskite $\mathrm{CsPbCl}_{3}$ Nanocrystals. RSC $A d v$. 2017, 7, 36722-36727.

(26) Zhou, Y.; Luo, J.; Zhao, Y.; Ge, C.; Wang, C.; Gao, L.; Zhang, C.; Hu, M.; Niu, G.; Tang, J. Flexible Linearly Polarized Photodetectors Based on All-Inorganic Perovskite $\mathrm{CsPbI}_{3}$ Nanowires. Adv. Opt. Mater. 2018, 6, No. 1800679.

(27) Liu, X.; Yu, D.; Cao, F.; Li, X.; Ji, J.; Chen, J.; Song, X.; Zeng, H. Low-Voltage Photodetectors with High Responsivity Based on Solution-Processed Micrometer-Scale All-Inorganic Perovskite Nanoplatelets. Small 2017, 13, No. 1700364. 